\title{
Pulsar science with the CHIME telescope
}

\author{
Cherry $\mathrm{Ng}^{1,2}$ on behalf of the CHIME Pulsar collaboration \\ ${ }^{1}$ Dunlap Institute, University of Toronto, \\ 50 St. George St., Toronto, ON M5S 3H4, Canada \\ email: cherry.ng@dunlap.utoronto.ca \\ ${ }^{2}$ Dept. of Physics and Astronomy, University of British Columbia, \\ 6224 Agricultural Road, Vancouver, BC V6T 1Z1, Canada
}

\begin{abstract}
The CHIME telescope (the Canadian Hydrogen Intensity Mapping Experiment) recently built in Penticton, Canada, is currently being commissioned. Originally designed as a cosmology experiment, it was soon recognized that CHIME has the potential to simultaneously serve as an incredibly useful radio telescope for pulsar science. CHIME operates across a wide bandwidth of $400-800 \mathrm{MHz}$ and will have a collecting area and sensitivity comparable to that of the 100-m class radio telescopes. CHIME has a huge field of view of $\sim 250$ square degrees. It will be capable of observing 10 pulsars simultaneously, 24-hours per day, every day, while still accomplishing its missions to study Baryon Acoustic Oscillations and Fast Radio Bursts. It will carry out daily monitoring of roughly half of all pulsars in the northern hemisphere, including all NANOGrav pulsars employed in the Pulsar Timing Array project. It will cycle through all pulsars in the northern hemisphere with a range of cadence of no more than 10 days.
\end{abstract}

Keywords. telescopes, instrumentation: interferometers, pulsars: general

\section{Telescope overview}

The Canadian Hydrogen Intensity Mapping Experiment (CHIME $\dagger$ ) is a radio telescope recently constructed at the Dominion Radio Astrophysical Observatory (DRAO) in Penticton, BC, Canada, and which is currently being commissioned. CHIME is composed of four cylindrical reflecting surfaces, each 100-m in length North-South (N-S) and 20-m wide East-West (E-W). This geometry provides an extremely wide effective field-of-view (FOV) of $\sim 120^{\circ}$ in N-S and $1.3-2.5^{\circ}$ (frequency dependent) in E-W, that is, a primary beam size of $\sim 250$ square degrees. With no moving parts in the structure of CHIME, it operates as a transit telescope, surveying the entire overhead sky each day as the earth rotates. On each of the four focal lines is a linear array of 256 dual polarization antennas. These antennas are made using printed circuit boards and have a clover-leaf shape that optimizes for the broad bandwidth of CHIME from 400-800 MHz (Deng et al. 2014). These antennas are arranged in a regular grid with a mean spacing of $0.3048 \mathrm{~m}$ in the $\mathrm{N}-\mathrm{S}$ direction and $22 \mathrm{~m}$ in E-W. We thus have a total of 2048 analog inputs and these are each amplified and brought through $50 \mathrm{~m}$-long co-axial cables to a digital F-X correlator.

In the correlator F-engine, 128 custom-made signal processing boards based on field programmable gate arrays (FPGAs; Bandura et al. 2016a, 2016b) digitize the analog radio signals collected and channelize the full bandwidth into 1024 frequency bins via a 4-tap polyphase filter bank (PFB), at a $2.56 \mu$ s cadence (see Table 1 ). In the X-engine, spatial correlation is performed in a GPU cluster that consists of 256 processing nodes each with 4 AMD Fiji GPUs, building on the Pathfinder system described in Recnik et al. (2015), Denman et al. (2015), and Klages et al. (2015). This GPU cluster also forms 10 dual-polarization tied-array beams, allowing us to track 10 pulsars at different locations

$\dagger$ www.chime-experiment.ca 
Table 1. The specifications of the CHIME/Pulsar project.

\begin{tabular}{ll|ll}
\hline & CHIME general parameters & \multicolumn{1}{l}{ Pulsar-backend specific parameters } \\
\hline Field of view & $120^{\circ}(\mathrm{N}-\mathrm{S}) ; 1.3-2.5^{\circ}(\mathrm{E}-\mathrm{W})=250 \mathrm{sq}$ degree & Number of phase bins & 1024 \\
Beam size & $0.26^{\circ}$ at $800 \mathrm{MHz} ; 0.52^{\circ}$ at $400 \mathrm{MHz}$ & Frequency resolution & $390 \mathrm{kHz}$ \\
System temperature & $\sim 50 \mathrm{~K}$ & Number of spectral channels & 1024 \\
Bandwidth & $400-800 \mathrm{MHz}$ & Output data bit depth & $16 \mathrm{bits}$ \\
Telescope latitude & $49^{\circ} 19.2 \mathrm{~m}$ & Number of polarizations & 2 \\
Telescope longitude & $-117^{\circ} 37.2^{\prime}$ & Pulsar data output rate & $67 \mathrm{Mbps}$ \\
\hline
\end{tabular}

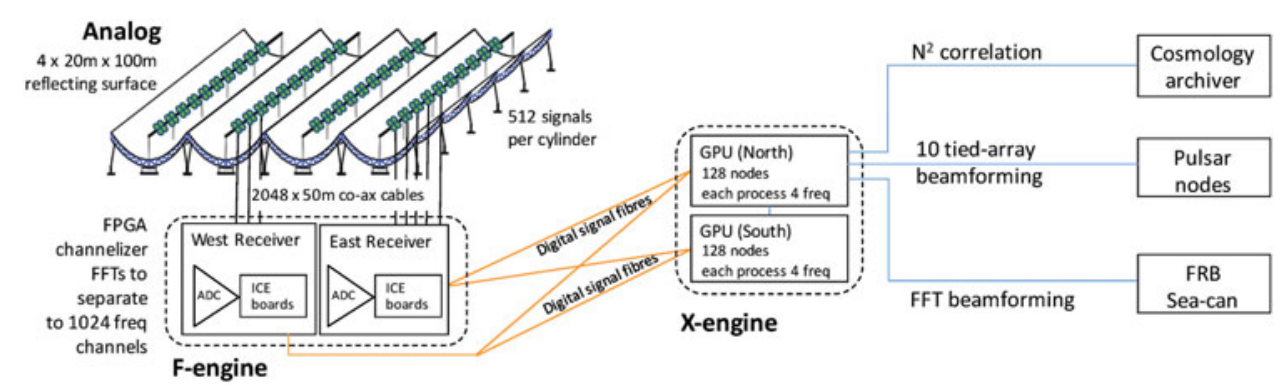

Figure 1. A system diagram of CHIME, showing the signal path from the analog system to the $\mathrm{F}$-engine, $\mathrm{X}$-engine, and the three independent data backends.

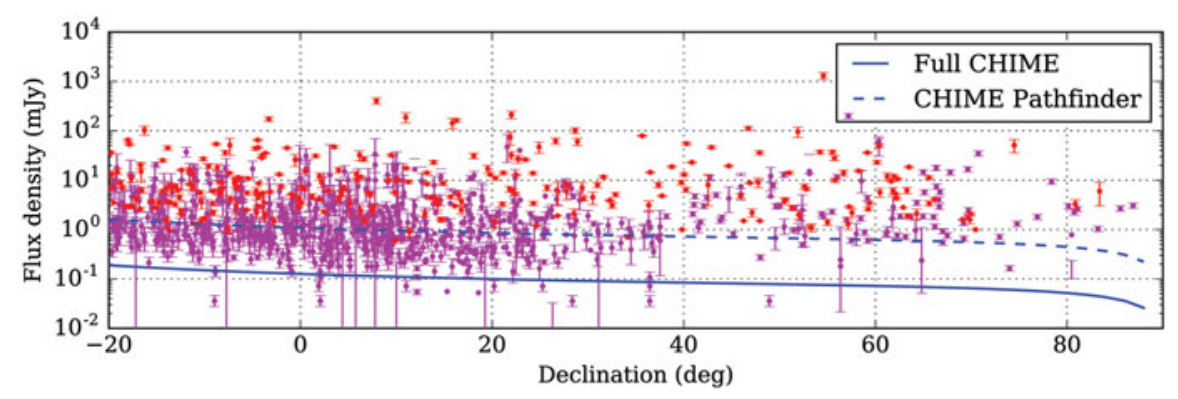

Figure 2. Minimum detectability of CHIME (solid line) and the Pathfinder (dashed line) as determined from the radiometer equation. Pulsars above $\delta=-20^{\circ}$ with published PSRCAT flux density at $600 \mathrm{MHz}$ are plotted as red dots and the error bars represent $1 \sigma$ uncertainties. For pulsars where only the flux density at another observing frequency is available, we scaled them to $600 \mathrm{MHz}$ using their respective spectral indices. These pulsars are plotted as magenta points.

simultaneously throughout the primary beam. A 10-node GPU-based pulsar backend will create folded pulsar archives with coherent dedispersion using the DSPSR software (van Straten et al. 2011). The output data will have 1024 frequency channels and 1024 phase bins with 4 stokes at a bit depth of 16 . We will have a read out cadence of $10 \mathrm{~s}$ which means a total output rate of $67 \mathrm{Mbps}$. See Figure 1 for a system diagram of CHIME.

\section{Telescope sensitivity}

CHIME will be able to observe pulsars down to a declination of about $-20^{\circ}$ (see Table 1). Because CHIME is a transit telescope, the maximum dwell time on each pulsar is a function of the source declination $(\delta)$. At the equator, this is approximately 10-15 minutes, while within the circumpolar region a source can be tracked for hours. Figure 2 shows the minimum detectable flux density of CHIME and the CHIME Pathfinder (Bandura et al. 2014), with the above factors taken into account. These limits are compared to 
the flux density of known pulsars above $\delta=-20^{\circ}$ published in the ATNF Pulsar Catalogue $\dagger$ (Manchester et al. 2005). It can be seen that CHIME should be able to detect the majority of the known pulsars in its visible sky.

\section{Science case}

Apart from the original science goal of mapping redshifted 21-cm hydrogen emission to study the Baryon Acoustic Oscillation signal at redshifts 0.8-2.5 (Bandura et al. 2014), as well as the search of Fast Radio Bursts (FRBs; see, e.g., Ng et al. 2017), a third CHIME backend is being deployed for high cadence pulsar timing, the focus of this proceeding.

\subsection{Pulsar timing for Gravitational wave detection}

CHIME plans to observe all visible NANOGravł pulsars daily to aid the effort of Pulsar Timing Array (PTA) experiments to detect Gravitational waves. Temporal dispersion measure (DM) and scattering variations will be monitored daily for each NANOGrav pulsar. The upper part of the CHIME band overlaps with the NANOGrav $820-\mathrm{MHz}$ observing band at the Green Bank Telescope (GBT). CHIME's wide bandwidth will provide a large 'lever arm' for measuring the DM as well as scattering time. In principle, the CHIME-measured, daily DMs could be used to reduce interstellar medium-related noise in NANOGrav data. Simulations suggest that this could mean an improvement in timing precision by a factor of at least 2 in at least half of the NANOGrav pulsars. However, Cordes et al. (2016) argue that DM variations might be effectively a frequency-dependent phenomenon, and hence the DM observed by CHIME at low frequencies would be distinct from that relevant at the higher PTA observing frequencies. CHIME will conduct regular simultaneous observations in conjunction with the GBT and the Arecibo Telescope at higher radio frequencies, which will help test models of the interstellar medium. CHIME will be of great use not only for NANOGrav but for all international PTA projects.

\subsection{Pulsars with time domain variability}

Not all pulsars have the same level of stability as those being employed in PTA experiments. Some pulsars exhibit time domain variability. For example, glitches in pulsars are discrete changes of the pulsar rotation rate thought to be a probe of the neutron-star interior. In one study, Espinoza et al. (2011) showed that thus far, 482 glitches have been observed from 168 pulsars $\uparrow$, although it is believed that the fraction of glitching pulsars as well as the frequency of glitch occurrence should be much higher. As another example, McLaughlin et al. (2006) discovered an entire class of pulsars with intermittent emission, now known as Rotating Radio Transients (RRATs). Telescope time on sky is the greatest factor limiting a thorough follow up and complete census of these types of variable pulsars. These sources will definitely benefit from a high observing cadence with CHIME.

CHIME also plans to perform daily monitoring of exotic objects such as high magnetic field $\left(\mathrm{B}>10^{13} \mathrm{G}\right)$ pulsars which might one day show magnetar-like outbursts (e.g. Archibald et al. 2016). Monitoring will also be conducted on X-ray magnetars that are currently radio quiet and any radio loud magnetar in the CHIME sky. Transitional MSPs (tMSPs; see, e.g., Archibald et al. 2009) swing between a rotation-powered state (radio emission) and an accretion-powered state (X-ray emission). CHIME will also be monitoring them to try and capture further moments of transition.

$\dagger$ http://www.atnf.csiro.au/people/pulsar/psrcat/

$\ddagger$ http://nanograv.org/

I See the online glitch table at http://www.jb.man.ac.uk/pulsar/glitches.html 


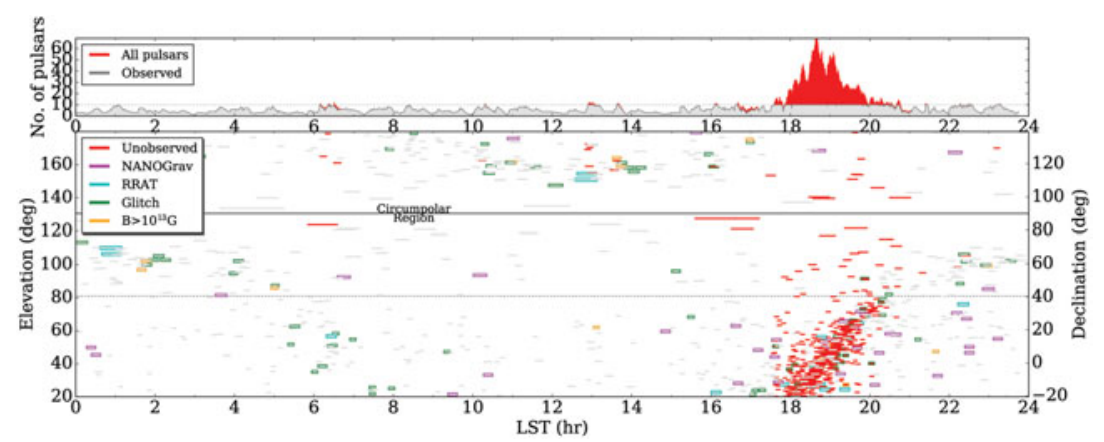

Figure 3. The lower panel shows all pulsars over the CHIME sky and the length of each bar represents the drift time. Over the course of one day, CHIME will have observed all the gray bars, i.e., some 700 pulsars. Only along the Galactic plane will we have unobserved pulsars (red bars), but these sources will be prioritized and observed within the next 10 days. The top panel shows the number of pulsars within the primary beam of CHIME at any given time.

\section{Observing strategy}

CHIME will have the ability to observe up to 10 pulsars simultaneously and with full polarization sensitivity, and is planned to operate $24 / 7$ as pulsars transit overhead. This is revolutionary and according to simulations (Figure 3), will allow for daily observation for roughly half of all North-visible pulsars, including all NANOGrav pulsars. CHIME will cycle through all pulsars in the northern hemisphere with a range of cadence no more than 10 days. CHIME is in a unique position to provide us with extremely high cadence pulsar observations and will surely lead to exciting new insights in the field.

\section{Acknowledgements}

We are very grateful for the warm reception and skillful help we have received from the staff of DRAO, which is operated by the National Research Council of Canada. We acknowledge support from the Canada Foundation for Innovation, the Natural Sciences and Engineering Research Council of Canada, the B.C. Knowledge Development Fund, le Cofinancement gouvernement du Quebec-FCI, the Ontario Research Fund, the CIfAR Cosmology and Gravity program, the Canada Research Chairs program, and the National Research Council of Canada. We thank Xilinx University Programs for their generous support of the CHIME project, and AMD for donation of test units.

\section{References}

Archibald, A. M. et al. 2009, Science, 324, 5933, pp. 1411-1414

Archibald, R. F. et al. 2016, ApJ (Letters), 829, L21

Bandura, K., et al. 2014, SPIE Astron. Telescopes+ Instrum., pp. 914522-914522

The CHIME Collaboration (Bandura, K., et al.) 2016a, J. Astron. Instrum., 05, 1641004

The CHIME Collaboration (Bandura, K., et al.) 2016b, J. Astron. Instrum., 05, 1641005

Cordes, J. M., Shannon, R. M., \& Stinebring, D. R. 2016, ApJ, 817, 16

Deng, M., Campbell-Wilson, D., \& CHIME Collaboration, T. 2014, ANTEM 16th Int'l Sym., 1

Denman, N. et al. 2015, Press. IEEE ASAP, pp. 35-40

Espinoza, C. M., et al. 2011, MNRAS, 414, 2, pp. 1679-1704

Klages, P., et al. 2015, Press. IEEE ASAP

Manchester, R. N., Hobbs, G. B., \& Hobbs, M. 2005, VizieR Online Data Catalog, 7245, 0

McLaughlin, A. M., et al. 2006, Nature, 439, 817

Ng, C., et al. 2017, XXXII International URSI GASS

Recnik, A., et al. 2015, Press. IEEE ASAP, pp. 57-61

van Straten, W. \& Bailes, B. 2011, PASA, 28, 1, pp. 1-14 